\title{
Fracture penis with urethral disruption \& scrotal hematoma: A case report
}

\author{
Nikhil Bhamare ${ }^{1}$, Shivaji Wadekar ${ }^{2}$, Sandesh Pawar $^{3}$,Sayalee Narwade ${ }^{4}$ \\ ${ }^{I}$ Department of General Surgery, Dr.S.C.Govt Medical college, Nanded, India. \\ ${ }^{2}$ Department of Urology, Dr.S.C.Govt Medical college, Nanded, India. \\ ${ }^{3}$ Department of General Surgery,N.D.M.V.P.Samaj's Medical college,Nashik,India. \\ ${ }^{4}$ Department of General Surgery, Dr.S.C.Govt Medical college, Nanded, India
}

\begin{abstract}
Penile fracture is a rare condition. Primarily it is a rupture of the corpus cavernosum that occurs when the penis is erect. The rupture can also affect the corpus spongiosum and the urethra.

We report a case of a 32 year old man who presented with acute penile pain, peno-scrotal swelling, perineal discoloration and the inability to pass urine after a blunt trauma during sexual intercourse one day back. In emergency surgery we found bilateral partial rupture of the corpus cavernosum with complete urethral and corpus spongiosum disruption. In the one year follow up the patient presented with normal erectile and voiding function.
\end{abstract}

Keywords: Fracture penis, urethral rupture, scrotal hematoma

\section{Introduction:}

Penile fracture is an entity of eminently clinical diagnosis, therefore the management of a penile fracture should not include any further investigation other than surgical exploration. ${ }^{1}$ The need for immediate surgery is emphasized, in order to avoid erectile failure and curvature, which are typical complications of conservative treatment. ${ }^{2}$ Many conditions can simulate fracture penis as dorsal vein tears in penis may mimic penile fracture. $^{3}$

\section{Case Report}

We report the case of a 32-year-old male who presented to the emergency department 24 hour following blunt injury of the penis during sexual intercourse. The patient reported a "tearing/popping" sensation, rapid detumescence, severe penile pain and frank blood per urethra after trauma. The patient was complaining of dribbling following the injury.

Physical examination demonstrated a tender and swollen penis with hematoma extending over the proximal penile shaft, scrotum and perineum. This examination suggested extension beyond Buck's fascia, but the hematoma appeared contained within Colles' fascia(Fig 1). "Rolling sign" was present bilaterally, palpated at the sites of tunical disruption about $3 \mathrm{~cm}$ from the base of the penis and was tender on examination. The scrotum was swollen and painful to palpation,

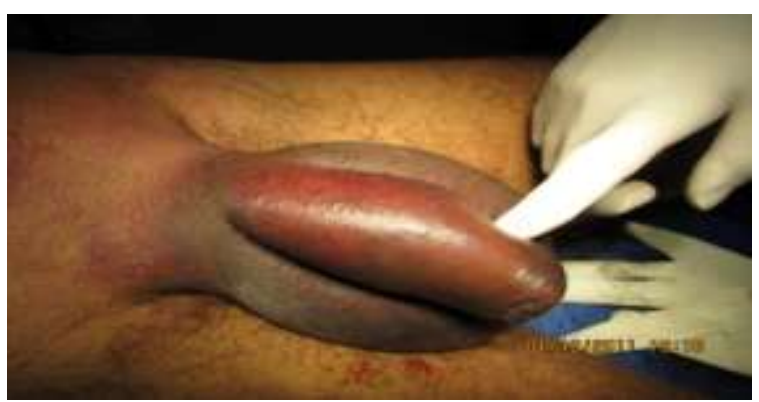

Fig. 1. Penile fracture with scrotal hematoma.

There was no known previous urologic history.

The patient was taken to the operating room for urgent exploration of suspected penile fracture with associated urethral injury. A 12-French Foley catheter was placed

A subcoronal circumferential incision was made and the penis degloved. Obvious disruption of Buck's fascia was evident; upon exploration, bilateral, $1-\mathrm{cm}$ transverse ruptures of the corpora cavernosa were discovered on the proximal penile shaft \& complete disruption of the urethra (fig.2). The defects were repaired with 3-0 vicryl sutures in a simple running fashion. (Fig. 3). Urethral breech was repaired over an 18-French 
catheter with 3-0 PDS suture with a simple interrupted end-to-end primary reanastomosis in a tension-free fashion (Fig.4).Two to three small scrotal incisions were taken \& hematoma was drained

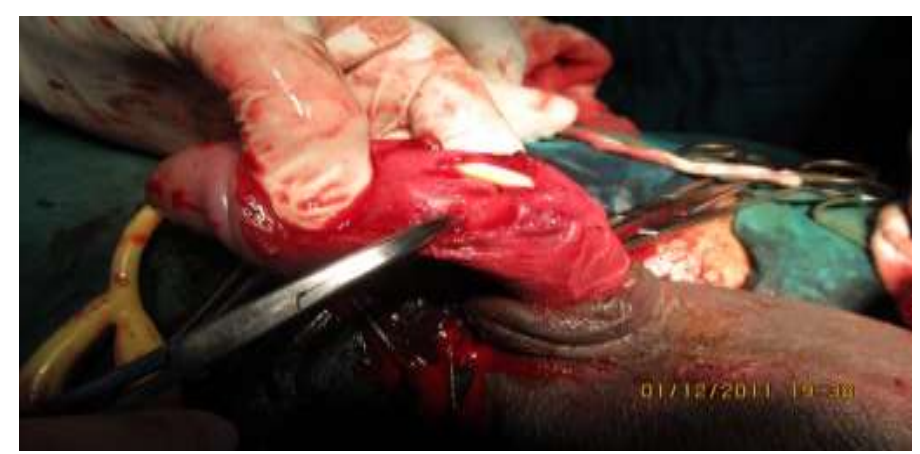

Fig. 2.Intraoperative photo demonstrating bilateral transverse rupture of the corpora cavernosa \& urethra

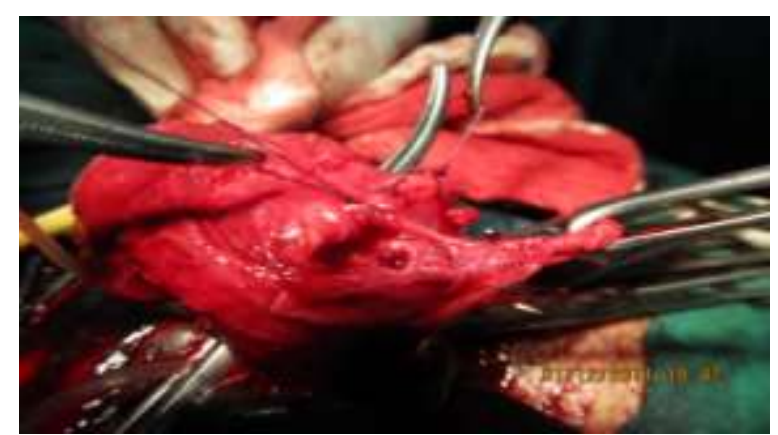

Fig.3. Photo showing primary reanastomosis of urethra.

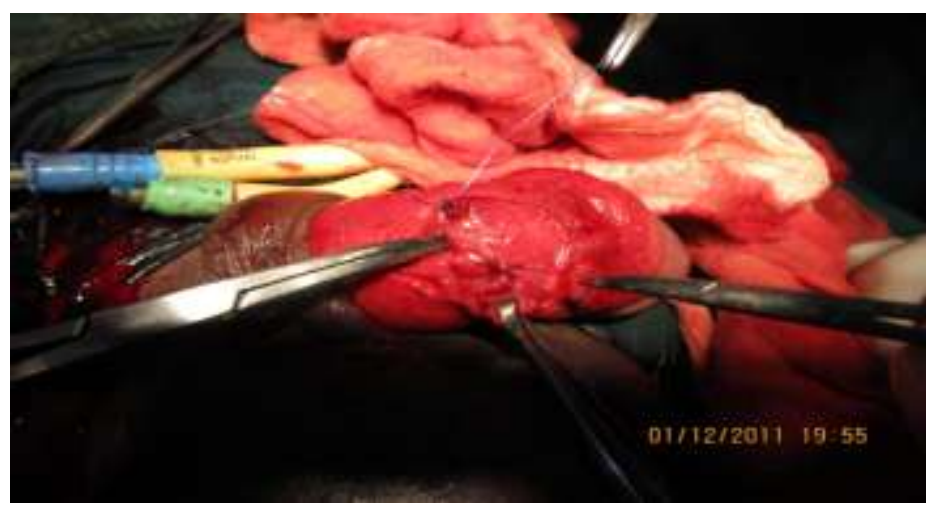

Fig. 4. Photo showing completed reanastomosis .

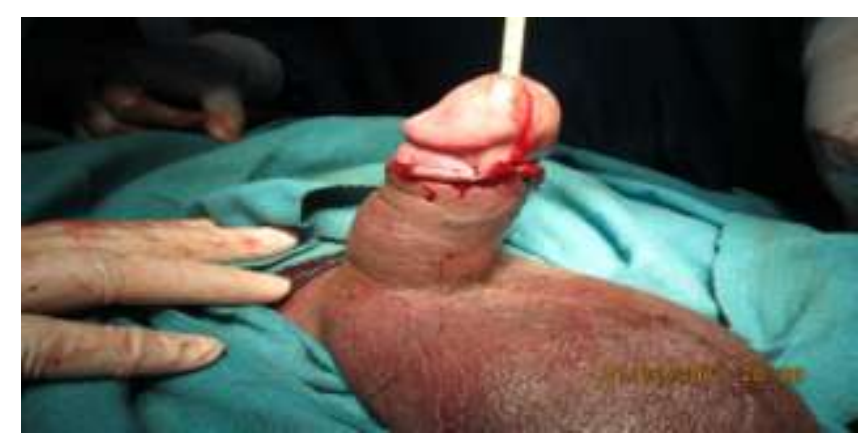

Fig. 5. Photo showing completed reanastomosis .

The patient recovered well in hospital and was discharged on postoperative day 2 on antibiotics with a Foley catheter to straight drainage and suprapubic catheter capped. The patient was advised to abstain from intercourse for at least 4 weeks. The patient underwent a RGU on postoperative day 21 , which was found normal with no contrast extravasation (Fig. 6). The urethral catheter was therefore removed, with the capped 
suprapubic catheter removed on postoperative day 28. Upon follow-up on day 28 , the patient reported normal voiding and satisfactory painless erections.

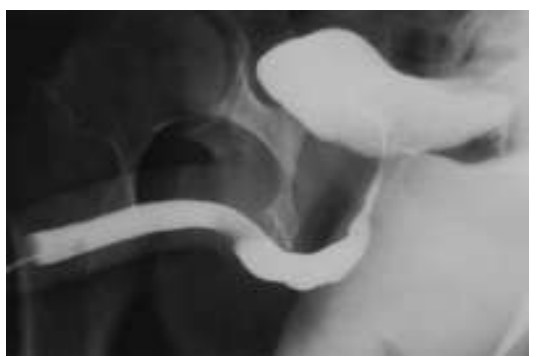

Fig. 6.Postoperative day 21: retrograde urethrogram demonstrating intact urethra.

III. Discussion

Fracture of the penis is a relatively rare condition, defined as the rupture of the tunica albuginea due to trauma to erect penis. ${ }^{4}$ Sporadic reports of penile fracture give the impression of a rare trauma but penile fracture is not so rare. ${ }^{5}$ The true incidence of penile fracture is not known even in western countries because it is under reported or hidden probably because of social embrassement and sociocultural characteristics, even it is reported to physicians it remains undiagnosed or mismanaged. ${ }^{6}$

Predisposing factors include excessive force at coitus or manipulation, fibrosclerosis of the tunica albuginea and chronic urethritis. Cause may vary from region to region depending on the socio-cultural characteristics, masturbation habits and indulgence in sexual activities. In the Western world the most common cause, accounting for about $30 \%-50 \%$ of cases, is over enthusiastic sexual intercourse. Of those, woman-on-top positions resulting in impact against the female pelvis or perineum and bending laterally are most common. In Middle Eastern countries the common cause is physical manipulation of the penis to remove an erection. ${ }^{5,7}$ Although, most injuries that result from masturbation come from forcibly handling an erection without care and fracturing it. Rarely it may occur while turning over in bed, forced bending, or hastily removing or applying clothing when the penis is erect in over enthusiasm.

In flaccid state it allows significant degree of deformation without any injury to the vital structures but in erected state it is vulnerable to blunt injury. As the penis changes from a flaccid state to an erect state, the thick tunica albuginea becomes very thin from $2 \mathrm{~mm}$ to $0.25-0.5 \mathrm{~mm}$; the tunica albuginea thins, stiffens, and loses elasticity and becomes easily fracturable.

Acute onset of pain, swelling, and ecchymosis of the penis during sexual intercourse indicate a penile fracture until proven otherwise. Fascia gives way and produces typical "butterfly-pattern" ecchymosis. Rolling sign can be demonstrated in fracture penis which is the movement of penile skin over the organized hematoma at the site of rupture of tunica albuginea. An entity called "false" penile fracture is a condition that closely mimics "true" penile fracture. Nonspecific dartos bleeding and avulsed superficial dorsal vein are causes. The absences of the "snap" and gradual detumescence both of which are distinguishing factors that suggest false fracture differentiates from true penile fracture but are not specific. Exploration is deemed necessary. Surgical treatment has an advantage and is safe, effective, restores erectile function satisfactorily for patients and partners and is highly recommended. ${ }^{5,7}$, Conservative management is associated with a high morbidity, and early surgical repair of the fracture is usually recommended in order to prevent complication. Non-operative treatment results in a $10 \%$ incidence of penile deformity and requires a substantial hospital stay .

Associated injuries included urethral rupture occurring in 10\% Delay in seeking treatment increases the complication rate non-surgical approaches result in 10\%-50\% complication rates including erectile dysfunction, permanent penile curvature, damage to the urethra, and pain during sexual intercourse. Immediate surgical repair in case of penile fracture is recommended for better functional outcome avoiding potential complications is associated with a low incidence of late complication. ${ }^{6,5,8}$ But there are proponents surgical repair even in case of delayed presentation.

\section{Conclusion}

Penile fracture is a true surgical emergency. Trauma to erect penis is essential for getting fracture penis. Awareness of mode of trauma and clinical features is all that required for diagnosis and no more investigation is desirable. Surgical exploration is ideal for its management. 


\section{References}

[1] Gontero P, Muir GH, Frea P. Pathological Findings of Penile Fractures and Their Surgical Management. Urol Int 2003; 71:77-82.

[2] Tejido Sánchez A, Martín Muñoz MP, Villacampa Abuá F, de la Morena Gallego JM, Suárez Charneco A, Leiva Galvis O. Surgical management of the penile fractures. Our experience. Actas Urol Esp 1999; 23:784-788.

[3] Bar-Yosef Y, Greenstein A, Beri A, Lidawi G, Matzkin H, Chen J. Dorsal vein injuries observed during penile exploration for suspected penile fracture. J Sex Med 2007; 4:1142-1146.

[4] Ateyah A, Mostafa T, Nasser TA, Shaeer O, Hadi AA, Al-Gabbar MA. Penile fracture: Surgical repair and late effects on erectile function. J Sex Med 2008; 5:1496-1502.

[5] Eke N. Fracture of the penis. Br J Surg 2002; 89:555-565.

[6] El Atat R, Sfaxi M, Benslama MR, Amine D, Ayed M, Mouelli SB, et al. Fracture of the penis: Management and long-term results of surgical treatment. Experience in 300 cases. J Trauma 2008; 64:121-125.

[7] Zargooshi J. Penile fracture in Kermanshah, Iran: The long-term results of surgical treatment. BJU Int 2002; 89:890-894.

[8] De Giorgi G, Luciani LG, Valotto C, Moro U, Praturlon S, Zattoni F. Early surgical repair of penile fractures: our experience. Arch Ital Urol Androl 2005; 77:103-105. 